\title{
Development of an Analogue Optical Link for the Front-end Read-out of the ATLAS Electromagnetic Calorimeter
}

\author{
B. Dinkespiler, M. Jevaud, C. Olivetto, O. Martin, \\ A. Mirea, E. Monnier S. Tisserant, M. Wielers \\ Centre de Physique des Particules de Marseille, CPPM \\ 163, Avenue de Luminy, Case 907, F-13288 Marseille Cedex 9, France \\ M.-L. Andrieux, J. Ballon, J. Collot, A. Patti \\ Institut des Sciences Nucléaires, ISN \\ 53, Avenue des Martyrs, F-38026, Grenoble Cedex, France \\ L.O. Eek, A. Go, B. Lund-Jensen, M. Pearce, J. Söderqvist \\ Royal Institute of Technology, KTH, Physics Dept. Frescati, \\ Frescativägen 24, S-10405 Stockholm, Sweden \\ J.-P. Coulon \\ Laboratoire de l'Accélérateur Linéaire, $L A L$ \\ Bât. 200, F-91405 Orsay Cedex, France
}

\begin{abstract}
We have developed an analogue optical data transmission system intended to meet the read-out requirements of the ATLAS liquid argon electromagnetic calorimeter. Eight-way demonstrators have been built and tested. The link uses arrays of VCSEL diodes as the optical emitters, coupled to a 70 metre long fibre ribbon to simulate the distance between the detector and the control room. The receiver is based around a custom-designed PIN photodiode array. We describe here the final results of laboratory tests on a demonstrator, laying stress on the VCSEL-to-fibre coupling issues, and the overall performance of the full link. A 9-bit dynamic range is achieved, with a $5 \%$ non-linearity.
\end{abstract}





\section{$1 \quad$ Preamble}

Our collaboration has already shown the feasibility of an optical system for the read-out of a preshower detector [1]. At that time, LED-based analogue links working at liquid argon temperature had demonstrated a large dynamic range, good linearity, radiation hardness, and had been successfully tested during test beams in September 1994 and 1995 [2]. Since then the replacement of the preshower by a coarser grained presampler, and the choice of warm preamplifiers and shapers for the first read-out stage of the electromagnetic calorimeter have made cold links redundant. However, we proceeded with our investigations on analogue optical links to try and develop a system capable of meeting the new requirements.

Today, the baseline scheme for the readout of the calorimeters makes use of digital optical links between the front-end boards and the read-out drivers. Analogue links could be considered as an interesting backup solution.

\section{Introduction}

Optical links offer some advantages over conventional copper links with coaxial cables:

- Compactness of the system. This is of prime importance when the system has to fit in a limited space. The front-end crates for the electromagnetic calorimeters readout system are located in the crack between the barrel and end-cap calorimeters cryostats, and at the rear end of the end-cap cryostats. Weight and dimension of copper cables and their associated connectics are a major obstacle to their being used in these zones.

- Immunity to electromagnetic interference. Constraining grounding rules will have to be applied if many sensitive electronic parts are to coexist in the front-end crates. Electronic systems could also be hampered by neighbouring systems, such as the solenoid power supply.

- Optical links allow long distance transmission, as they show little signal attenuation; simple copper links are ruled out for distances greater than a few tens of metres.

- Low power consumption and thermal dissipation. It is a common concern in the frontend area of the detector, given the proximity to a large cryogenic system, as a small increase in power dissipation has strong consequences on the cooling budget.

The main motivation for transmitting analogue signals with an optical link is the possibility to read-out all channels downstream of the preamplifiers, allowing sensitive electronics, such as analogue pipelines and ADCs, to be moved to the trigger cavern $70 \mathrm{~m}$ away from the detector, or $200 \mathrm{~m}$ away, in the control room. In either location, the electronics would be freely accessible for maintenance, whereas the radiation level in the front-end position could raise reliability issues. Also, an analogue optical system does not add any source of pickup noise.

The performance requirements for an analogue link are as follows:

- A large dynamic range.

Signals coming from the calorimeter have a dynamic range in excess of 16 bits. The preamplifiers are followed by a three gain shaper, with gains approximately in the ratio $1 / 10 / 100$ [3]. They reduce the range to 12 bits, which comfortably covers the expected signal range, and allows a safety margin to cope with hypothetical coherent noise. When 
we started our project, the use of a four-gain system was contemplated, which made a less stringent requirement of 10 bits for the link.

- Very good linearity, at the level of one percent over the entire dynamic range, would be dictated by the performance level of the shapers. The requirement could be reduced to a few percent if a fit or look-up table proved to be efficient.

- Large bandwidth, in excess of $100 \mathrm{MHz}$, to prevent any degradation of physics signals.

- Very good reliability, especially for the emitter part situated in the front-end crate. Components will have to withstand yearly doses of $80 \mathrm{~Gy}$ and neutron fluence of $2 \cdot 10^{12}$ $\mathrm{n} / \mathrm{cm}^{2}$, during 10 years of $\mathrm{LHC}$ operation.

- As mentioned above, the power dissipation has to be low.

The next section gives an overview of the solution we investigated, then in the fourth section the properties of the emitter components are presented. In the fifth section we show how the connection to the optical fibres was performed for our demonstrator links. The sixth section is a brief overview of the receiving end of the link, which has already been thoroughly described in a previous note [4]. Finally, section 7 shows the laboratory tests of the whole link.

\section{Presentation of the proposed link}

Our wish was to achieve a high degree of integration, as there would be one link per channel of the electromagnetic calorimeters, i.e. about 190000 . This led us to choose arrays of optical emitters connected to fibre ribbons. For the reception part the level of integration is identical. The principle of the analogue optical link is described in figure 1 . Downstream of the 3-gain preamplifier and shaper system, the signal is a voltage whose amplitude is proportional to the physical signal (energy deposited in a calorimeter cell). The voltage at the output of the shapers would be used to modulate the laser. At the receiver end the optical signal is converted back to electrical current by arrays of PIN photodiodes, then into a measurable voltage by transimpedance amplifiers.

Only the purely analogue part of the the link is studied in this note. In the conditions of use in ATLAS, a system of fast gain selection, such as a fast comparator, would have to be inserted between the shapers and the optical link. The transmission of the digital 2-bit gain information is not addressed here. This could be achieved either by a composite signal, or by adding a separate channel. The second solution would probably be preferred, because a composite link would be too complicated and would require a larger bandwidth. A coding scheme would have to be studied, to optimise the bandwidth of the digital signals to be transmitted.

\section{The emitter}

Two types of semiconductor device produce light: Light Emitting Diodes (LEDs), and Laser Diodes (LDs).

LEDs are cheap, and do not raise the eye-safety problems associated with the use of powerful lasers. On the other hand, their strongly divergent light cone favours coupling to expensive large core optical fibres (typically $200 \mu \mathrm{m}$ ). Their threshold current, which is high (10-100 $\mathrm{mA})$, raises the issues of power dissipation and system reliability. The semiconductor 
tracker [5] plan to use a coding scheme to balance zeros and ones (so-called 'Non-Return to Zero' protocol), and to switch off the LEDs when no information is transmitted.

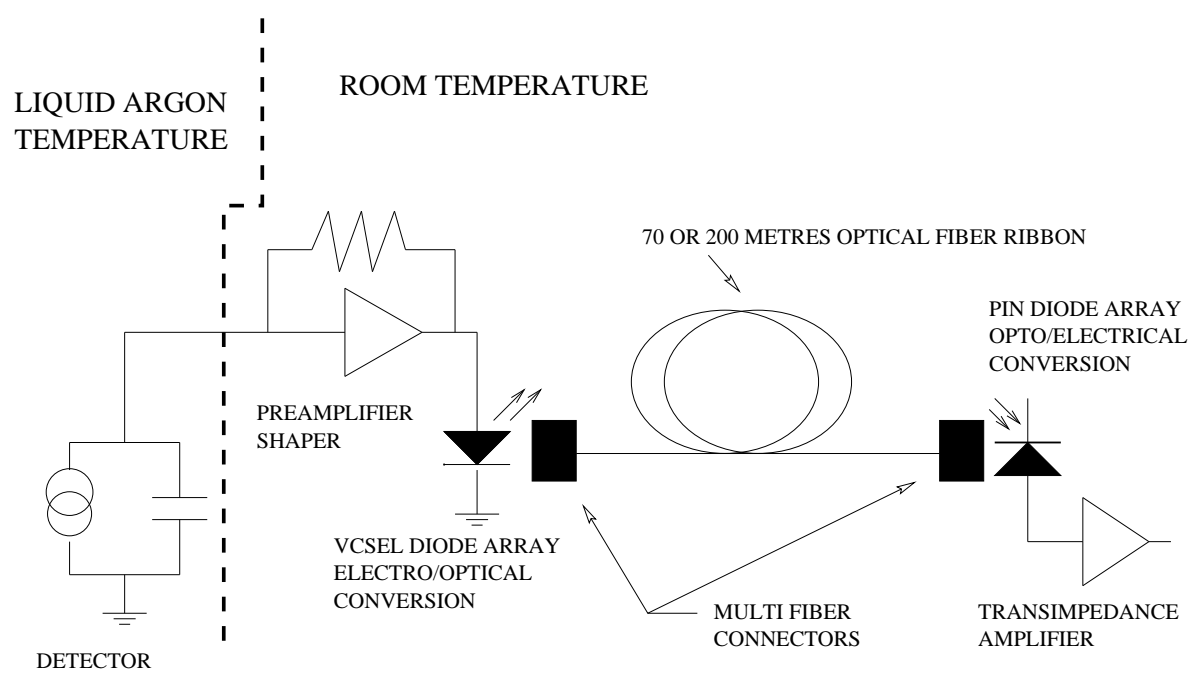

Figure 1: Schematic diagram of an analogue optical link for the read-out of the liquid argon calorimeters.

Laser diodes are usually much more powerful than LEDs (a few $\mathrm{mW}$ compared to less than $100 \mu \mathrm{W})$. Emitters with sophisticated structures such as Distributed Bragg Reflectors (DBR) or Multiple Quantum Wells (MQW), have excellent optical performance in terms of linearity, but are expensive. Their light emission is very elliptical, and astigmatic, which complicates the connection to the fibres.

As LEDs and LDs are edge-emitting devices, this reduces the level of integration, as no array of components can be made. This fact led us to investigate a newly available type of emitter, the Vertical Cavity Surface Emitting Laser diode (VCSEL). VCSELs appeared nearly two decades ago [6], but only started emerging from laboratories since the early 1990 s to find their way to commercial applications ${ }^{1)}$. VCSELs are ideal for high-speed interconnect links. They emit a low-divergent, circular beam which has a high coupling efficiency into optical fibres. VCSELs have a low current threshold relative to other lasers, typically below $4 \mathrm{~mA}$, which makes them easy to drive. Devices with sub-mA threshold are becoming available. Their small active area provides them with a very low capacitance and high modulation speed, at the level of tens of $\mathrm{GHz}$.

The vertical structure of VCSELs allows fabrication processes of the same kind as those found in microelectronics. Every step leading to the final product can be done at the wafer level, and one or two-dimensional arrays of components can be obtained. Tests carried out on the wafer make it easier to identify individual elements with homogeneous performance level before any packaging is done, thus reducing the production costs. Yields of $99 \%$ across a typical 3-inch wafer have been announced [7], and yields of more than $90 \%$ are routinely achieved for a high volume commercial production [8].

\footnotetext{
1) one of the first was Motorola's Optobus (now discontinued), and now there are many available Gigabit transceivers based on VCSELs (HP, Honeywell, Methode, ...)
} 
Sandia National Laboratories ${ }^{2)}$ provided us with arrays of VCSELs for our development. We performed preliminary probe tests of the devices. As the manufacturer proceeded with his developments, successive generations of lasers were sent to us, some of which better matched our needs. A photograph of a Sandia VCSEL array is shown in figure 2. The lasers are laterally oxidised VCSELs, with a wavelength of $850 \mathrm{~nm}$.

The first demonstrator modules we built (July 1997) used VCSELs of a "conventional" type, i.e. the semiconductor substrate is n-type GaAs, and the upper mirror stack ends with a p-type layer.

For the next demonstrator modules (end 1997), we benefited from a new structure developed by Sandia, on which the substrate is p-doped, and the last mirror is n-doped. These VCSELs have an inverted polarity compared to the previous ones. As shown in figure 3 , they have a current threshold at the sub-mA level, and a maximum output power slightly below $4 \mathrm{~mW}$. They are supposed to have a lower resistance compared to the conventional noninverted structures [9]. From the voltage vs current curve in figure 3, a dynamic resistance of about $100 \Omega$ at a $2 \mathrm{~mA}$ bias can be inferred. Lower threshold current and resistance usually have positive consequences in terms of noise and power dissipation. In the following sections, we will present setups and results for the tests of a demonstrator based on the latter type of VCSELs, which was more thoroughly studied. Results from the first generation demonstrator can be found in $[10]$.

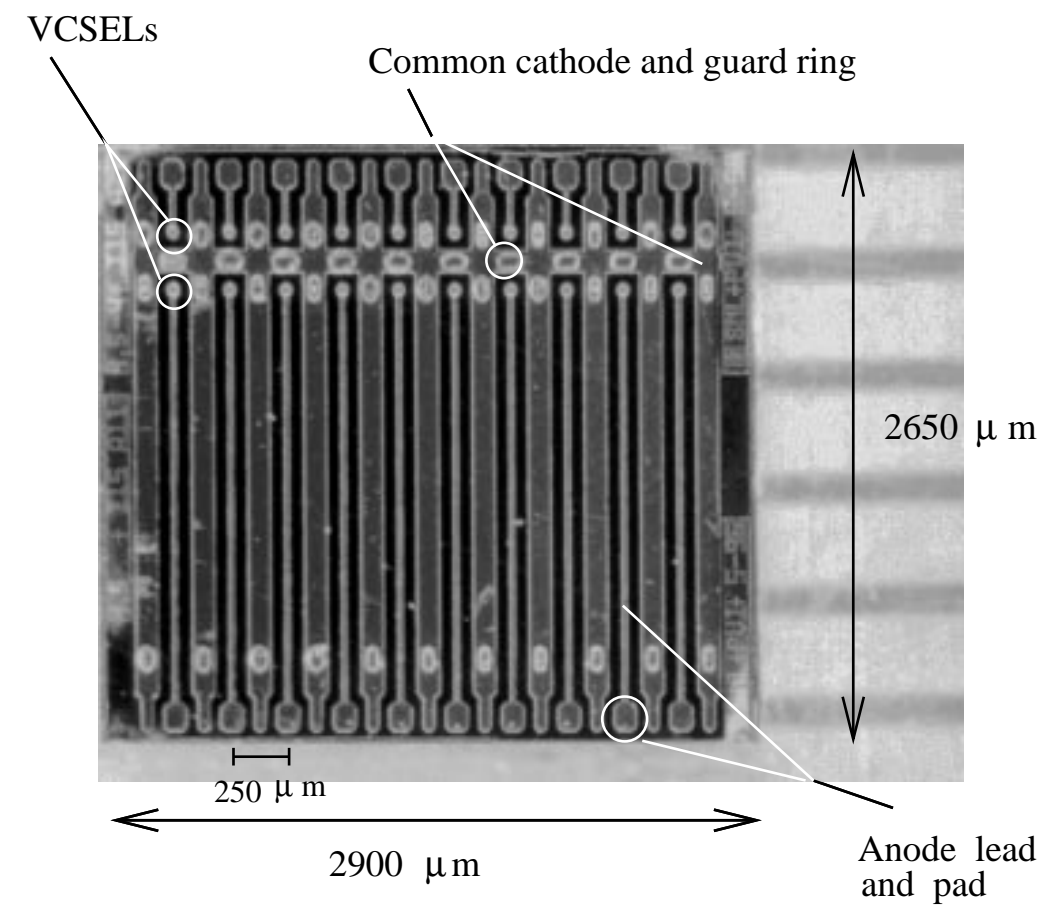

Figure 2: Photograph of a Sandia VCSEL array. There are 20 lasers per chip, 10 with long anode leads and 10 with short leads. For convenience, only the 8 central lasers with long leads are bonded and consequently used in a demonstrator module.

2) Team led by K.L. Lear, now at MicroOptical Devices, Inc., Albuquerque, New Mexico 


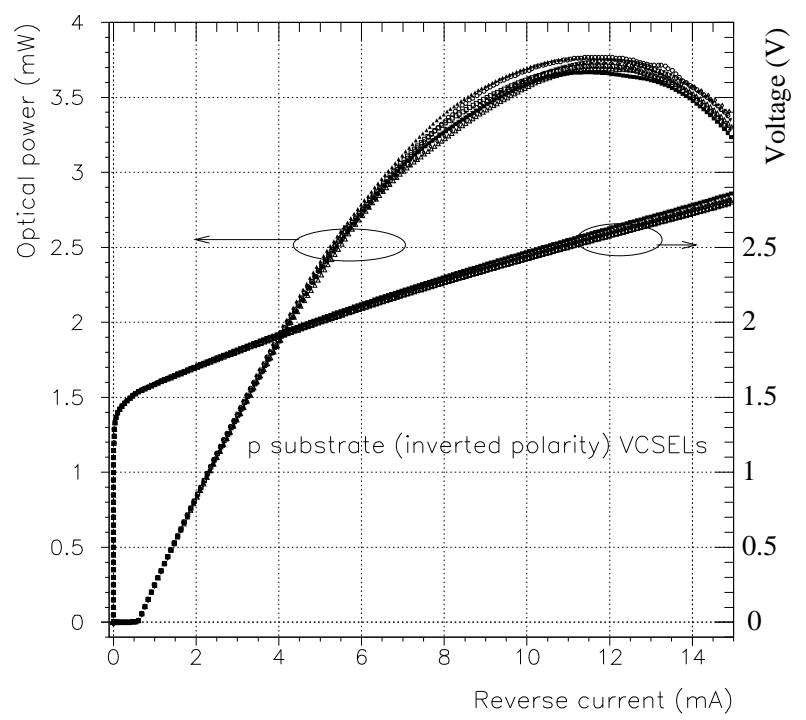

Figure 3: Free space $c w$ optical power vs current and voltage vs current characteristics of eight lasers of a n-mirror-up VCSEL array. The thermal roll-off (saturation of the output power at large bias currents) is visible.

Tolerance to radiations of various emitters has been studied by the group involved in the read-out of the inner detector; more details can be found in their note [11]. Among the devices tested about 140 were VCSELs provided by Sandia. About a hundred of them were of the same type as those we used in the first demonstrator.

Degradation by irradiation is due essentially to displacement damage, when atoms of the lattice are hit by incident particles. This phenomenon, known as Non-Ionising Energy Loss (NIEL), introduces defects which can act as recombination centres in the forbidden bandgap of the semiconductor. Radiation-induced non-radiative recombination centres decrease the minority carrier lifetime $\tau$, and subsequently the quantum efficiency of the optical emitter, as the Relative Light Output (RLO) can be directly related to the ratio $\frac{\tau}{\tau_{0}}[11]$, $(\mathrm{RLO})^{\frac{3}{2}}=\frac{\tau}{\tau_{0}}$, where $\tau_{0}$ is the carrier lifetime before irradiation.

In the case of a VCSEL, in the lasing regime the minority carrier lifetime is dominated by stimulated emission, and is several orders of magnitude lower than for LEDs. One can thus expect the VCSEL to be intrinsically more tolerant to radiations than LEDs, as a higher fluence is required to significantly change the ratio $\frac{\tau}{\tau_{0}}$. On the other hand, under the laser threshold, $\tau$ is governed by spontaneous emission, like in the LEDs, and the threshold current is expected to increase after irradiation.

The tests carried out proved that VCSELs are really radiation hard, as devices irradiated with fluence up to $4 \cdot 10^{14} \mathrm{n} / \mathrm{cm}^{2}$ emit as much light as before the irradiation, after a short annealing of a few days. What is most remarkable, devices operated in pulsed mode during the irradiation showed very little degradation at all. We could then expect some sort of self-annealing in an ATLAS environment if VCSELs are continuously operated above their threshold current, which would be acceptable, knowing the low power dissipation of these devices.

After annealing these components were submitted to an aging cycle with the equivalent 
of up to 44 years in the ATLAS SCT environment [11]; the failure rate proved to be less than $1 \%$ for a batch of VCSELs of the same sort as those we used for our first demonstrator. We have no data concerning inverted-polarity VCSELs, which were not available at the time of the irradiation tests.

Otherwise few data are available regarding the reliability of VCSELs. For the most common proton implanted devices, a Mean Time To Failure (MTTF) of up to $3 \cdot 10^{7}$ hours is claimed [8,12], which is of the same magnitude as for conventional edge emitting devices. Concerns about the oxidised structures were legitimate at the early stages of their development, when lifetimes as short as a few minutes were reported [13]. These devices used pure AlAs, which inferred mechanical stresses by thickness changes after oxidation, and led to failure. No such behaviour was noticed with structures based on AlGaAs oxides [14]. Hopefully the mastering of this technology is soon to occur and life expectancies as long as for conventional edge emitting devices will be common.

\section{Connection to the fibre}

Our will was to develop a compact link without resorting to expensive technologies such as integrated optics. The laser arrays were received as bare chips, then were mounted on a standard DIL-sized ceramic. For the first 8-channel demonstrator modules, a custom connector socket was developed within our collaboration. The emitters were actively aligned to the 8-fibre ribbon terminated by a MT connector ${ }^{3)}$ (the principle is shown in figure 4 ). A view of the DIL ceramic, with its connector socket installed, is shown in figure 5 . The fibre was of the standard multimode $62.5 / 125 \mu \mathrm{m}$, graded-index type, provided by Acome ${ }^{4)}$. This Germanium-doped fibre has a typical attenuation of $2.8 \mathrm{~dB} / \mathrm{km}$ at $850 \mathrm{~nm}$, and a numerical aperture of 0.275 .

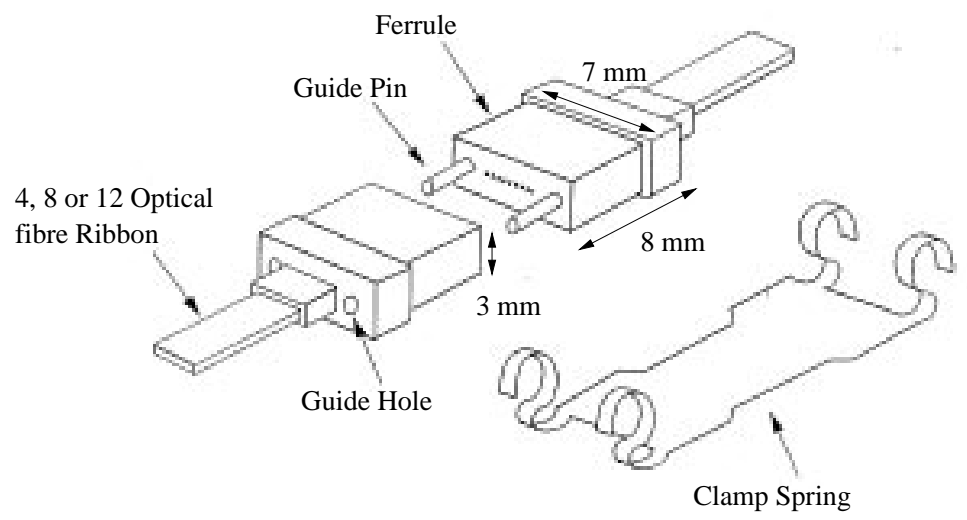

Figure 4: Principle of the MT multi-fibre (4 to 12) connector. In a row the fibres are $250 \mu \mathrm{m}$ apart, which matches the spacing between the VCSELs on an array. In our project, we used 8-way fibres and connectors.

With our socket design the entry face of the fibre could not be made closer than about $50 \mu \mathrm{m}$ above the laser. Preliminary alignment tests (figures 8 and 9 ) made us confident that

3) Distributed in Europe by Europtics Ltd, Helsby, Cheshire, UK.

4) ACOME, cable division, Paris, France. 
the coupling efficiency would be sufficient.

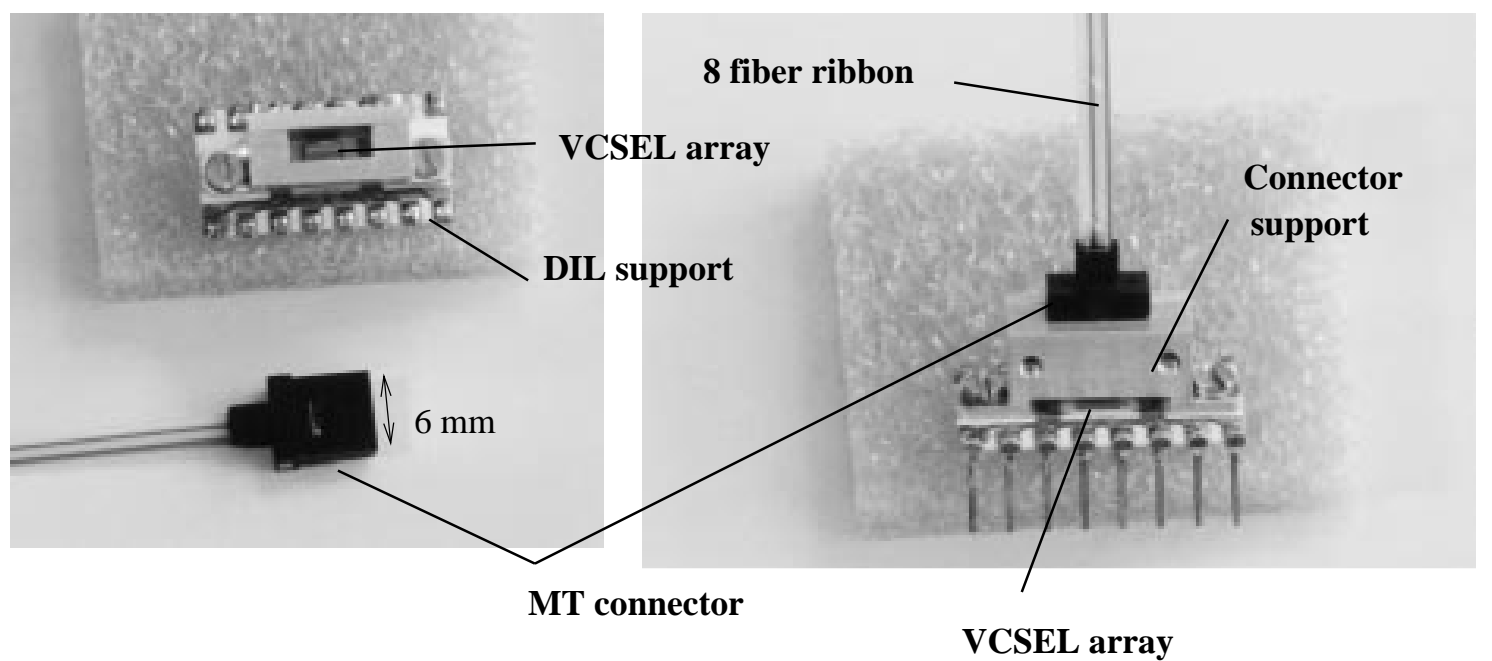

Figure 5: Photograph of the packaging designed for the first demonstrators. The VCSEL array is visible at the centre of the ceramic, and above the connector socket with the 8-fibre MT-connector.

Figure 6 shows the experimental setup for the measurements of continuous wave (static) emission. The free space emission was characterised with the VCSEL array mounted on a probe station, and the light was collected with a large area optical head connected to an optical multimeter. After the VCSELs were connected to a fibre ribbon, the optical head was replaced by a MT-to-ST8 fibre fan-out, to adapt to the ST-type entry of the optical multimeter. A sketch of the fan-out is visible in figure 7.

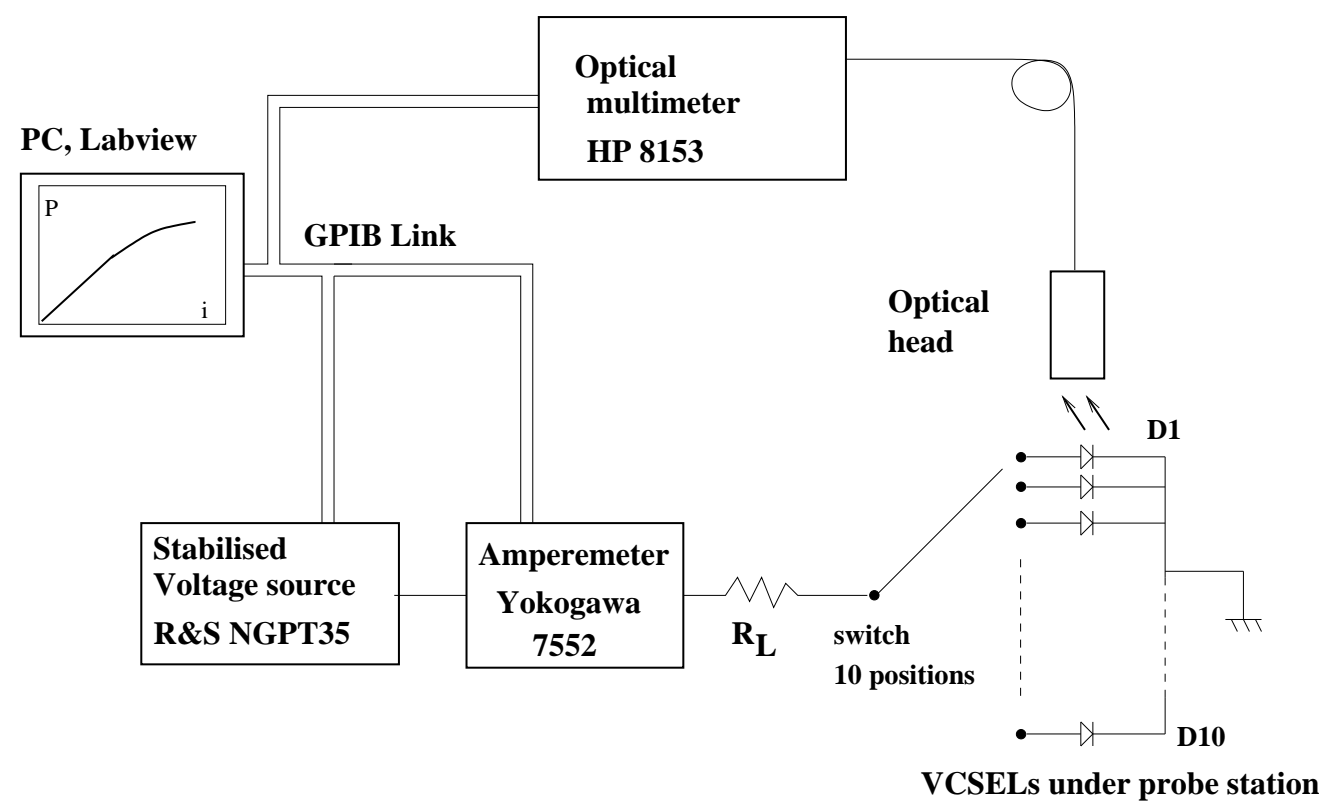

Figure 6: Setup for the static tests of the VCSEL diodes. The principle of the probe tests is shown here. 


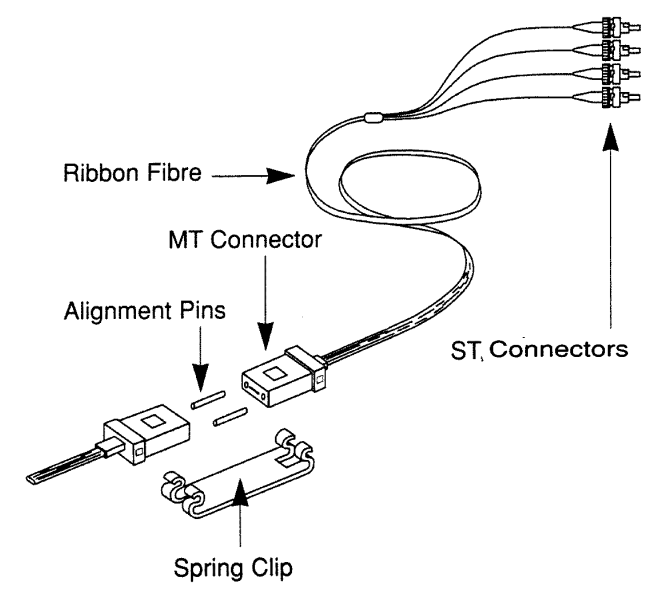

Figure 7: MT-to-multi ST fan-out used to measure individually the light output of the VCSELs after connection.

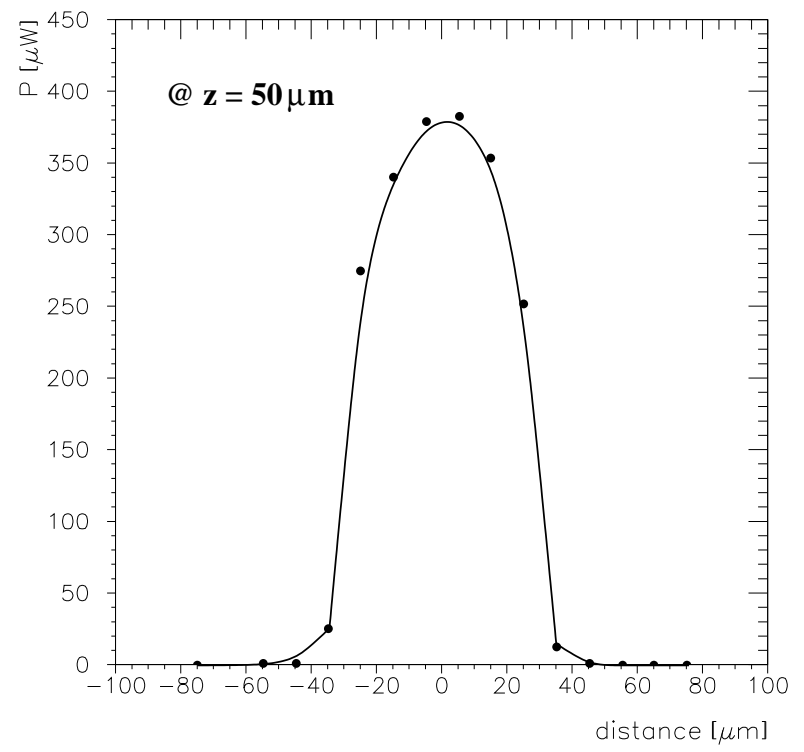

Figure 8: Preliminary alignment test, showing the lateral tolerance when connecting a VCSEL to a fibre. The fibre has a core $62.5 \mu \mathrm{m}$ in diameter, the VCSEL is $10 \mu \mathrm{m}$ wide with a light cone divergence of $\sim 13^{\circ}$, and the distance along the $z$ axis is $50 \mu \mathrm{m}$ between the fibre and the laser. The curve shown is not the result of a fit; the non-gaussian profile is probably caused by the multimode behaviour of the laser. 

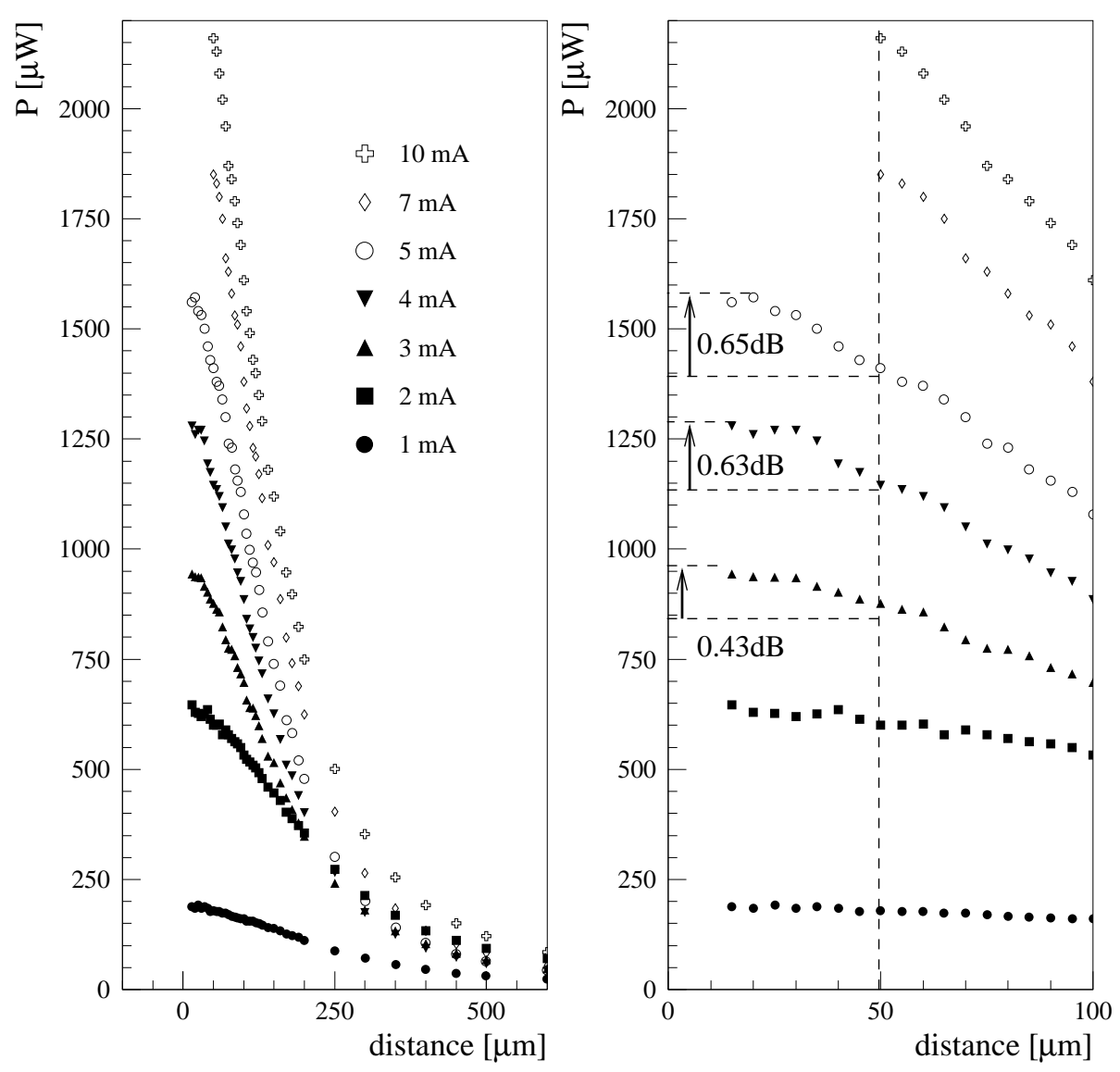

Figure 9: Insertion loss between the VCSEL and the fibre as a function of the distance along the $z$ axis. Left: on this large-scale plot the $1 / z^{2}$ behaviour is visible; right: at $z=50 \mu \mathrm{m}$, the loss is well below $1 \mathrm{~dB}$ for typical values of the current bias.

The resulting insertion losses for the entire link is presented in figure 10 for the latest demonstrator module. A loss at the level of $1 \mathrm{~dB}$ is observed when coupling a $1 \mathrm{~m}$ long fibre pigtail to the VCSELs, and another $1 \mathrm{~dB}$ when attaching $70 \mathrm{~m}$ of fibre to the pigtail. In this case, $\sim 0.3 \mathrm{~dB}$ are expected to originate from the fibre length, whereas the typical loss specified for a MT-to-MT connection is $0.3 \mathrm{~dB}$. Part of the remaining loss (less than $0.4 \mathrm{~dB}$ ) may be a measurement bias with the optical multimeter, as a large area optical head is used for the free space measurements, and a separate channel with additional MT and ST connections are used to measure the light output of connected VCSELs.

This rather simple design performed well within our expectancies in terms of coupling efficiency, as the maximum power transmitted through several connections is still high. In conclusion the overall attenuation of about $2 \mathrm{~dB}(\sim 3 \mathrm{~dB})$ can be expected for a realistic link $70 \mathrm{~m}(200 \mathrm{~m})$ long. Of course the dynamic range will be limited by the (max. output)/noise ratio, as will be seen later. A noise a the level of a few tenths of $\mu \mathrm{W}$ would be tolerated to meet the requirements, if the maximum power transmitted is $\sim 1 \mathrm{~mW}$. 

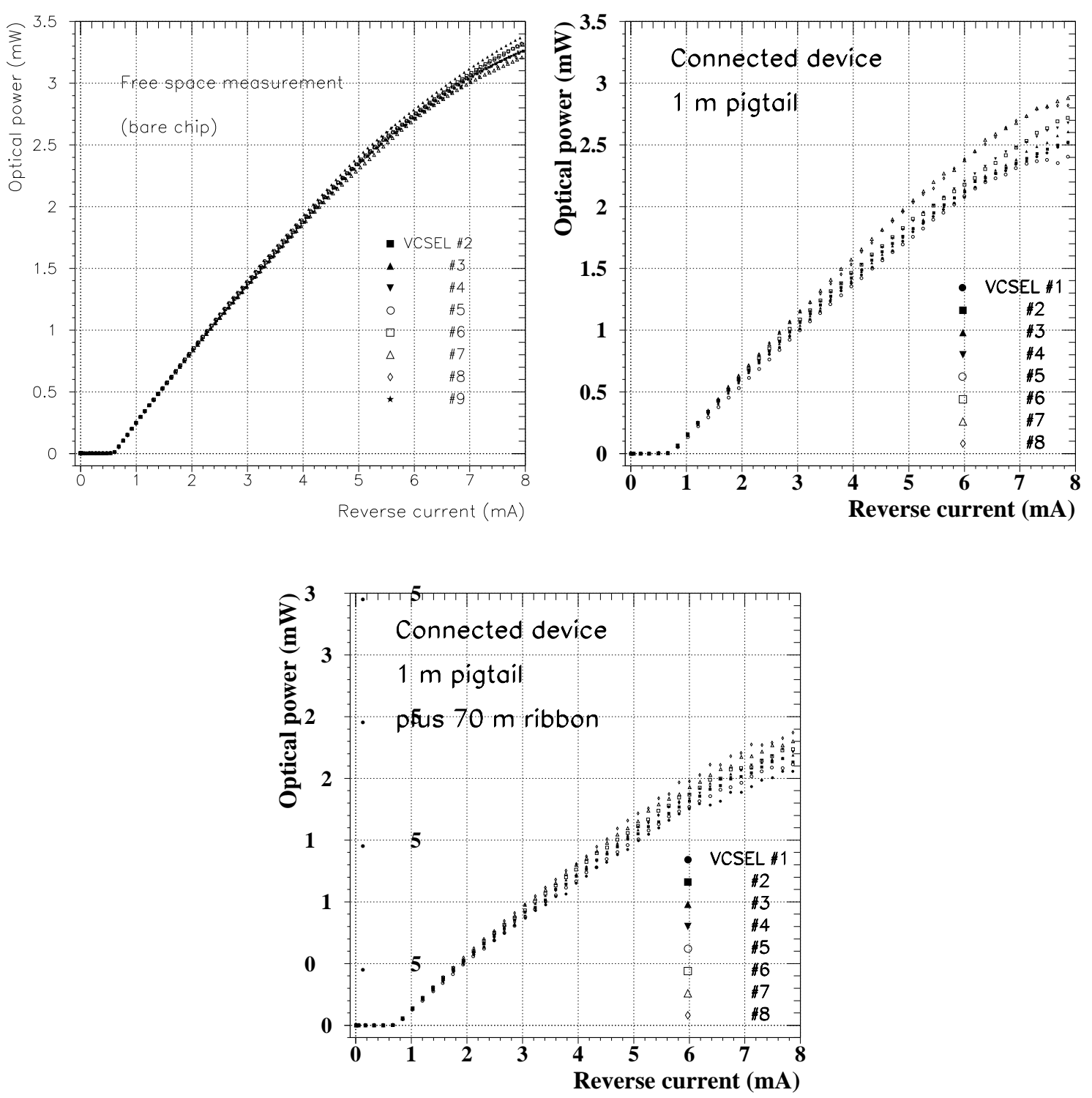

Figure 10: cw Light vs Current characteristics of all channels of the latest demonstrator showing the insertion losses (top left: bare chip, free space emission; top right: emitter array coupled to a $1 \mathrm{~m}$ fibre pigtail; bottom: after subsequent coupling to a $70 \mathrm{~m}$ ribbon). The total insertion and connection losses visible between the first and third plots are below $2 \mathrm{~dB}$.

\section{The receiver}

The conversion of the optical signal into electrical signal is based on silicon PIN photodiodes ${ }^{5)}$. The receiver stage of the link has already been described in detail in a note [15].

The MT connector from the incoming fibre ribbon was passively aligned and coupled to the PIN diode array, with the help of two guide pins. As for the emitter part, this head-on coupling is simple and cheap, because it includes no $\mathrm{V}$-groove or light guide. The active

5) provided by AME, Horten, Norway. 
surface of each photodiode, $175 \times 330 \mu \mathrm{m}^{2}$, is large compared to the diameter of the fibre core, which makes the alignment easy and the optical coupling efficient. A coated cover-glass was placed on the PIN array, both to protect the active surfaces and to reduce the reflections which could cause cross-talk or feedback noise. With this cover-glass the responsivity of the photodiodes is $0.5 \mathrm{~mA} / \mathrm{mW}$. The minimal reverse voltage (for 8 diodes) is $30 \mathrm{~V}$, above which the capacitance per detector element is less than $1 \mathrm{pF}$ (and constant). The typical value we used for the tests of the demonstrators was $60 \mathrm{~V}$. With a reverse bias voltage of $100 \mathrm{~V}$, a rise-time of $0.7 \mathrm{~ns}$, measured with a pulsed laser, and optical crosstalk below $0.1 \%$ were recorded.

The PIN diodes were connected to a custom-designed transimpedance amplifier. The transimpedance value is $2.7 \mathrm{k} \Omega$, yielding a maximum output of $2.5 \mathrm{~V}$ for optical signals of $2 \mathrm{~mW}$. The transimpedance amplifier response to square impulses had rise and fall times of $3.6 \mathrm{~ns}$.

A photograph of a receiver unit is shown in figure 11 .

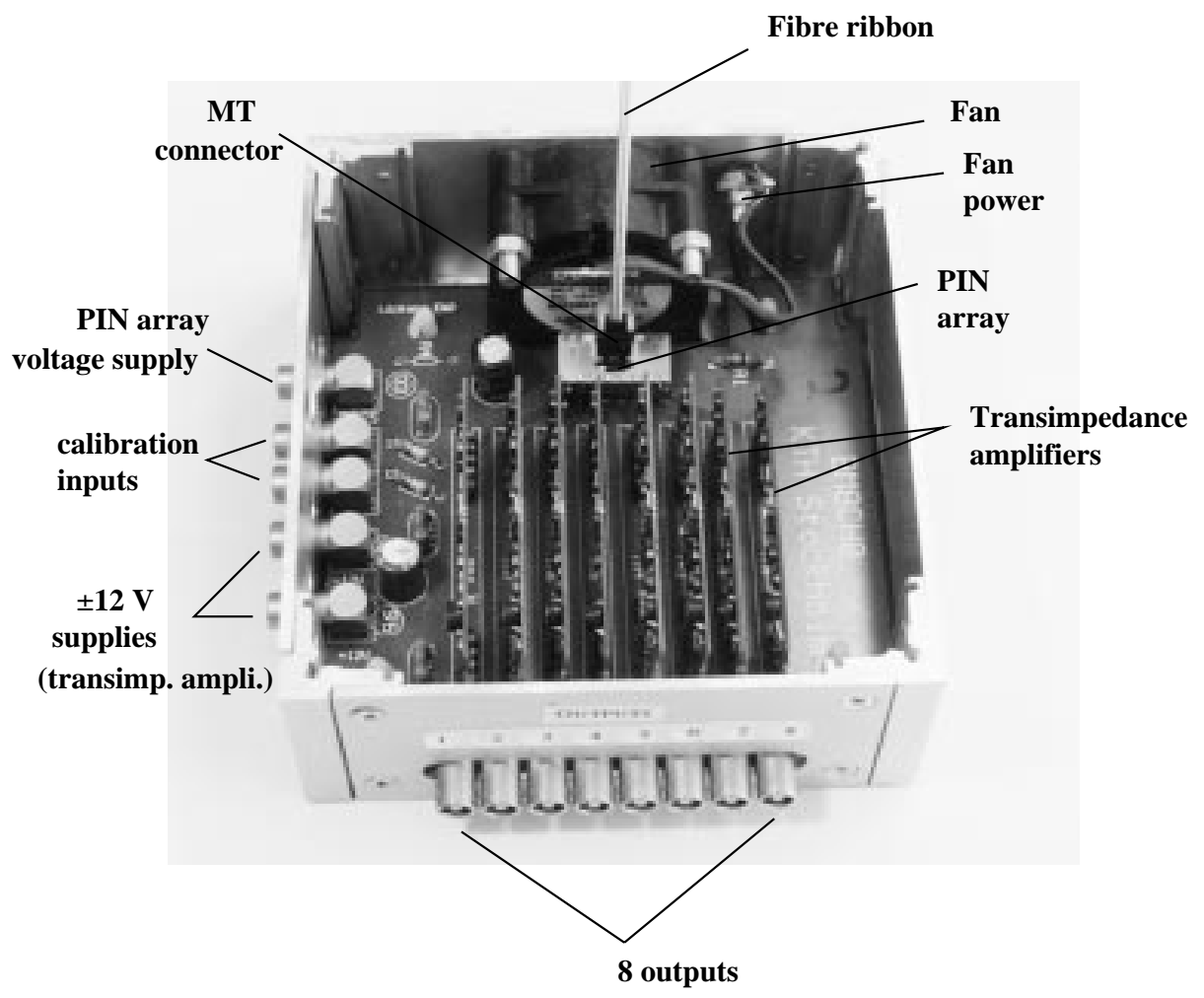

Figure 11: A photograph of a prototype receiver unit (lid removed).

The complete receiver unit was tested with bipolar signals with a $20 \mathrm{~ns}$ shaping time, as would be typical for LAr calorimeter signals. A noise level of $45 \mu \mathrm{V}$ (equivalent to $1.5 \mathrm{pA} / \sqrt{\mathrm{Hz}}$ ) was measured. The bandwidth exceeds $100 \mathrm{MHz}$ and the settling time of the response is small ( $<10 \mathrm{~ns})$. The total dynamic range of the receiver unit exceeds 13 bits, with a differential non-linearity of less than $1 \%$ over the entire range. 


\section{$7 \quad$ Performance of a complete demonstrator link}

\subsection{Noise measurements}

Observations of the analogue electrical signal in the frequency domain revealed resonances compatible with optical feedback noise. This phenomenon has been known since the early times of development of communication systems with semiconductor lasers [16]. Although it is more a concern for monomode lasers, it is also likely to occur in multimode transmissions.

When a laser diode is connected to a fibre, two kinds of noise (low- and high-frequency) are generated due to the injection of reflected waves from the input and output of the optical fibre. The low-frequency noise is caused by random interferences between the laser internal field and the reflected waves, and has a spectral range of tens of $\mathrm{kHz}$. It makes the laser intensity unstable. The latter noise, also called the self-coupling effect, directly disturbs the signals over the $0-4 \mathrm{GHz}$ range. It is caused by a resonance of the quantum shot noise of the laser diode in the external cavity formed by the laser mirror and the reflection point at the fibre end, as symbolised in figure 12 .

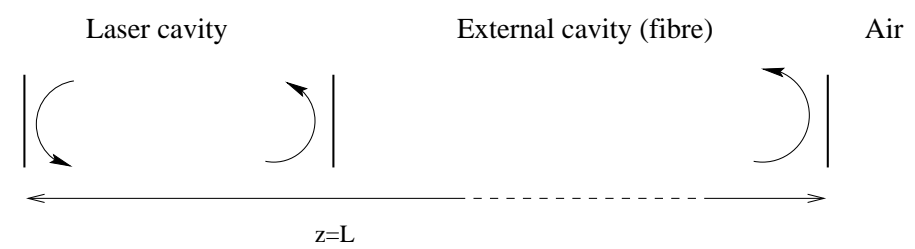

Figure 12: Principle of a laser cavity coupled to an external optical feedback. The length of the resulting cavity includes that of the laser (which is negligible compared to the fibre's).

We have not clearly noticed any low-frequency noise, and the continuous mode characteristics of the lasers remained stable after coupling to the fibre ribbon. However, spread among the 8 VCSELs static characteristics which seems to occur in figure 10 could be a manifestation of low-frequency noise.

On the other hand, a self-coupling effect seemed to show up. Reference [16] gives the expression of the resonance frequencies $f_{\text {res }}$ as a function of cavity length (or fibre length

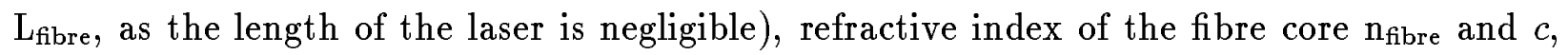
the speed of light in vacuum:

$$
\mathrm{f}_{\mathrm{res}}=\frac{\mathrm{k} \cdot c}{2 \mathrm{n}_{\mathrm{fibre}} \cdot \mathrm{L}_{\mathrm{fibre}}} \text { with } \mathrm{k}=0,1,2, \ldots
$$

We have experimented with several combinations of fibre lengths, including the $1.15 \mathrm{~m}$ long pigtail coupled to the VCSEL array, then adding another $20 \mathrm{~m}$ or $70 \mathrm{~m}$ fibre ribbon. Figure 13 shows the noise spectra obtained with the different fibre configurations, and the laser biased in DC mode (first three plots). Equation (1) is confirmed at the uncertainty level of the measurement, with corresponding resonances at 85, 4.6 and $1.4 \mathrm{MHz}$, respectively.

In monomode applications one is often encouraged to use angled connectors to help to keep the optical reflections at an acceptable level. This has not proved efficient in our case, as can be seen on the bottom noise spectrum curve in figure 13, obtained by coupling a VCSEL array to a $1.2 \mathrm{~m}$ pigtail with both MT connectors angled. 

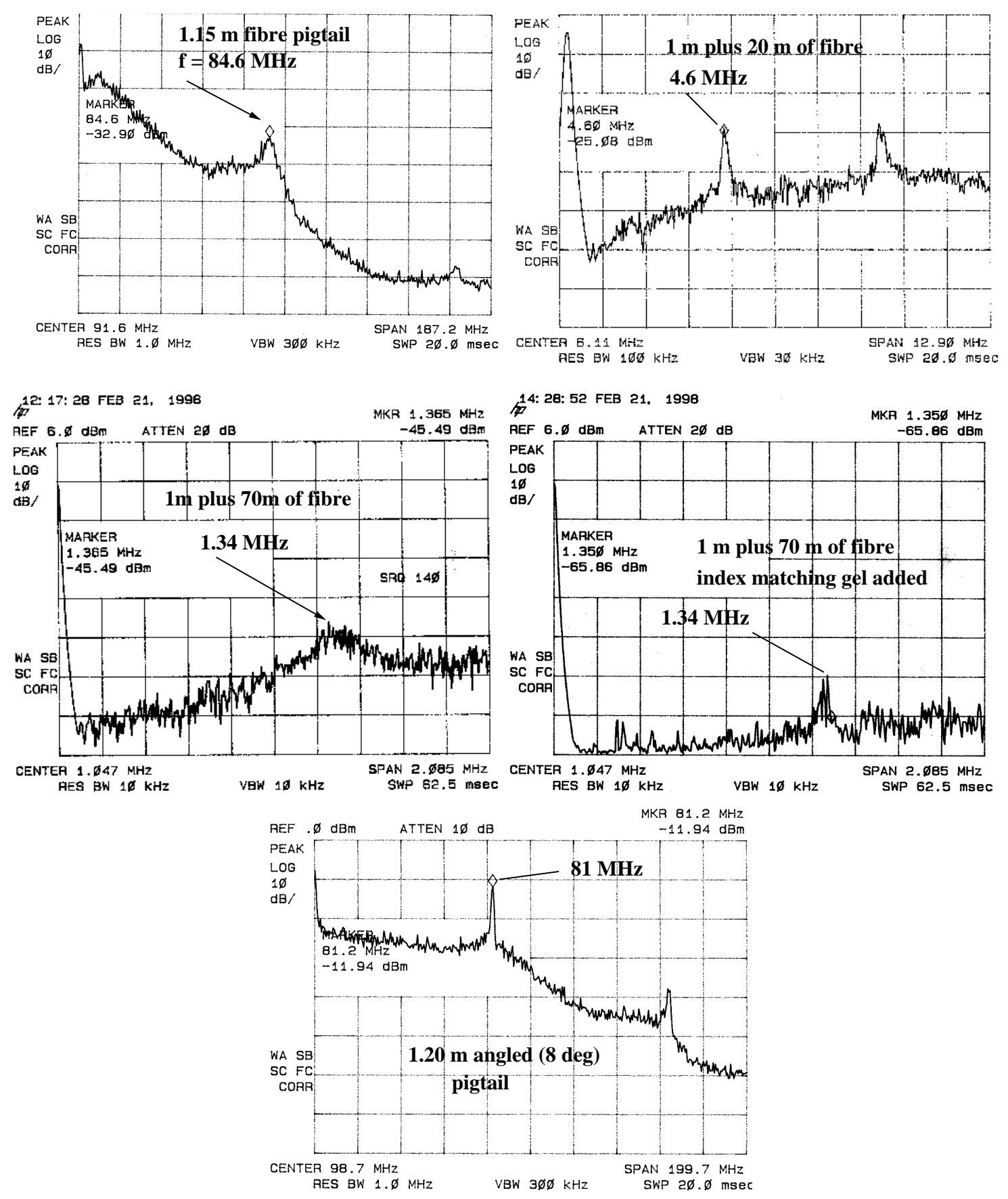

Figure 13: Noise spectra showing optical reflection noise due to feedback from the fibres. The spectra were obtained while biasing the VCSELs in DC mode, by feeding the output electrical signal of the receiver unit to a spectrum analyser. The relative level (dBm units) is plotted as a function of the frequency. The peak at $0 \mathrm{~Hz}$ is an artifact of the spectrum analyser. The first 3 plots show the resonances from cavities of $1 \mathrm{~m}$ (top, left), $21 \mathrm{~m}(20+1)$ (top, right) or $71 \mathrm{~m}$ (middle, left). Middle, right: index matching gel placed between two MT connector helped to reduce the noise. Bottom: with a $1 \mathrm{~m}$ fibre patch cord with both MT connectors angled polished at $8^{\circ}$, reflection noise is still high. 
Index matching gel has also been tried, with some success, as the noise level could be reduced when coating the interfaces of the MT connectors between the $1 \mathrm{~m}$ pigtail and the $70 \mathrm{~m}$ ribbon. For comparison, the spectra without and with index matching gel are placed next to each other (figure 13, middle curves). But this solution was not explored any further, because the gel coating proved to be unstable, and is not supposed to be radiation hard.

The total noise of the link was measured by feeding a low-noise amplifier (gain of 100) with the electrical signal from the output of the receiver stage. It was then sent either to an oscilloscope where $\mathrm{V}_{\mathrm{AC} \text { rms }}$ was observed, or to a spectrum analyser.

The conversion from $\mathrm{mV}$ (on the oscilloscope) to $\mathrm{mW}$ is straightforward, knowing the transimpedance value $(2700 \Omega)$ and the response of the PIN diode $\left(0.5 \mathrm{~A}^{-\mathrm{W}^{-1}}\right)$ :

$$
\mathrm{P}(\mathrm{mW})=\frac{\mathrm{V}(\mathrm{mV})}{2700 \times 0.5}
$$

Quantitative NEP measurement for one channel of the demonstrator is shown in figure 14 , in two cases. Without any index matching gel (open circles), the NEP is above $1 \mu \mathrm{W}$ and increases with bias current, showing an unstable behaviour. The dynamic measurements were carried out later in these conditions. With index matching gel (triangles), the noise remains stable and at the $0.5 \mu \mathrm{W}$ or lower.

With this demonstrator we adopted a bias point of $1.5 \mathrm{~mA}$ for the dynamic measurements, which seemed sufficiently above laser threshold to be in a linear regime, and corresponded to a minimum in the noise level.

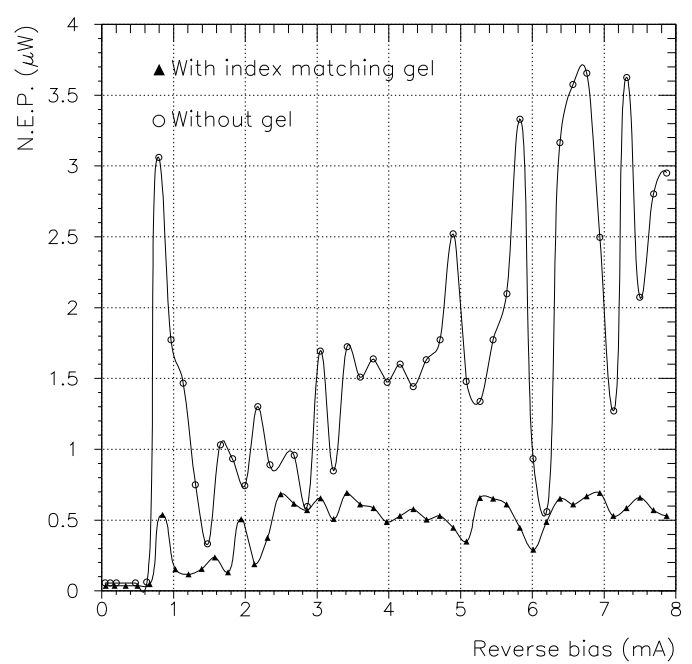

Figure 14: Noise (NEP) measurement for VCSEL $n^{0} 1$ of the latest demonstrator, with (triangles) or without (open circles) index matching gel at the connection between the $1 \mathrm{~m}$ pigtail and the $70 \mathrm{~m}$ ribbon. Use of gel was not adopted because of its instability with time.

\subsection{Setup for dynamic measurements}

The performance of the complete optical link in dynamic mode was measured by injecting voltage modulations to each emitter channel and by observing the voltage output at the corresponding receiver end. A sketch of the setup is presented in figure 15 . 


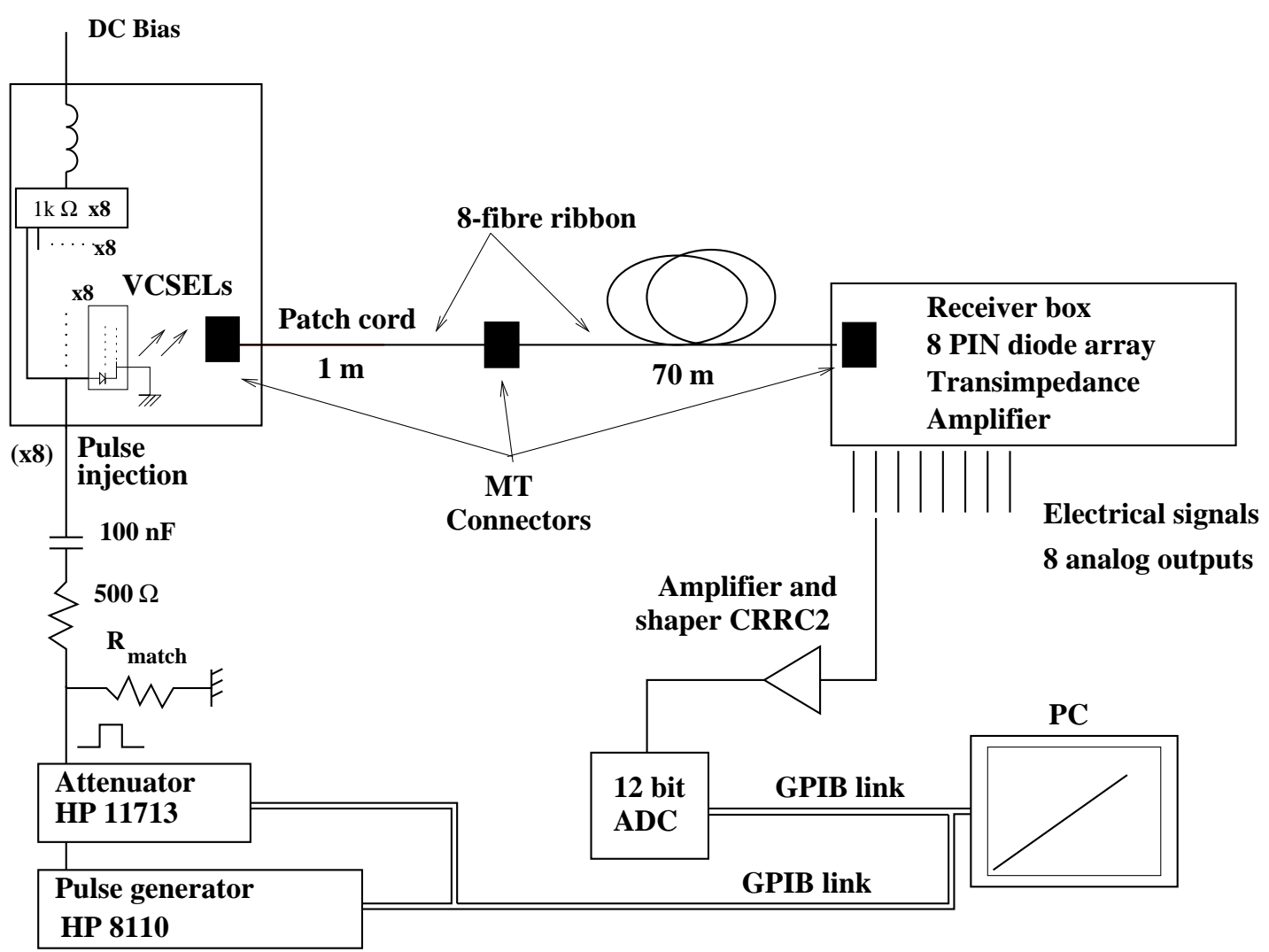

Figure 15: Experimental setup for the measurement of the dynamic response of the analogue optical link.

The VCSELs were biased above the threshold current, at $1.5 \mathrm{~mA}$ per laser diode. Independently of the DC bias, each laser was AC-coupled, as is visible in figure 15. A high frequency pulse generator connected to a high precision GPIB-programmable attenuator provided square voltage pulses (rise- and fall-time limited to $2 \mathrm{~ns}$ by the generator).

The overall dynamic range was split in $20 \mathrm{~dB}$ subranges (a factor 10 in voltage pulse, i.e. more than 3 bits), with overlaps between them so as to avoid any systematic effect caused by range switches of the read-out chain.

Figure 16 shows the response to a 200 ns square pulse $1.5 \mathrm{~V}$ in amplitude corresponding to a current pulse of $1 \mathrm{~mA}$ through a VCSEL. The value of the current impulse was checked with a probe through a $100 \Omega$ resistor added in series with the VCSEL, and by adjusting the amplitude of modulation to get the same response as in figure 16 .

For the dynamic measurements the analog output from the receiver stage was filtered by a bipolar amplifier with a $20 \mathrm{~ns}$ shaping time and $\sim 85 \times$ gain. The data acquisition was performed with a 12 bit ADC (read by GPIB bus). The ADC had to be triggered by a falling edge. In order to get well separated lobes and to be able to trigger on the negative one, the square pulse had to be chosen longer than $100 \mathrm{~ns}$, as can be seen in figure 17 . Non-linearities of the attenuator and of the amplifier-shaper-ADC were corrected for.

In this way the duty cycle of the link and the modulation frequency are low and not representative of the ATLAS-like conditions. But this test has to be considered as a first step 
in the evaluation of the dynamic performance of an analogue optical link based on VCSELs. Moreover, the properties of the signal were not degraded when using $10 \mathrm{~ns}$ long pulses with a period of $50 \mathrm{~ns}$ (see figure 18, to be compared with figure 16). The fall-time and amplitude are almost identical in spite of shorter pulses and higher duty cycle. This is not surprising, as most VCSELs are known to show cut-off frequencies of several $\mathrm{GHz}$, and as the bandwidth of the receiver stage exceeds $100 \mathrm{MHz}$.

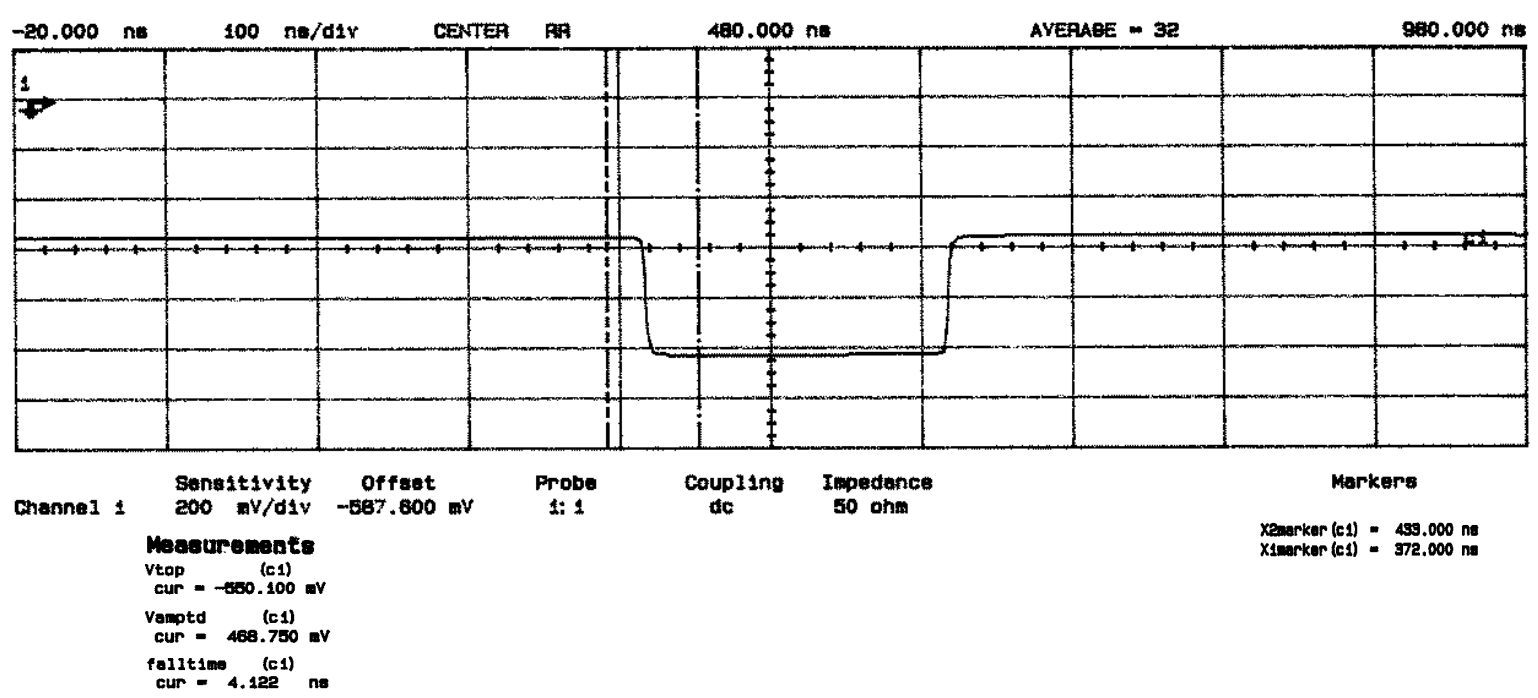

Figure 16: Response of a channel of the optical link demonstrator to a square modulation $200 \mathrm{~ns}$ in length and $1.5 \mathrm{~V}$ in amplitude. The current modulation in the laser is of $1 \mathrm{~mA}$ in this case. The fall time of the response is $4.1 \mathrm{~ns}$, and amplitude is $\sim 470 \mathrm{mV}$. (scales on the oscilloscope: $100 \mathrm{~ns} /$ div horizontally and $200 \mathrm{mV} /$ div vertically.)

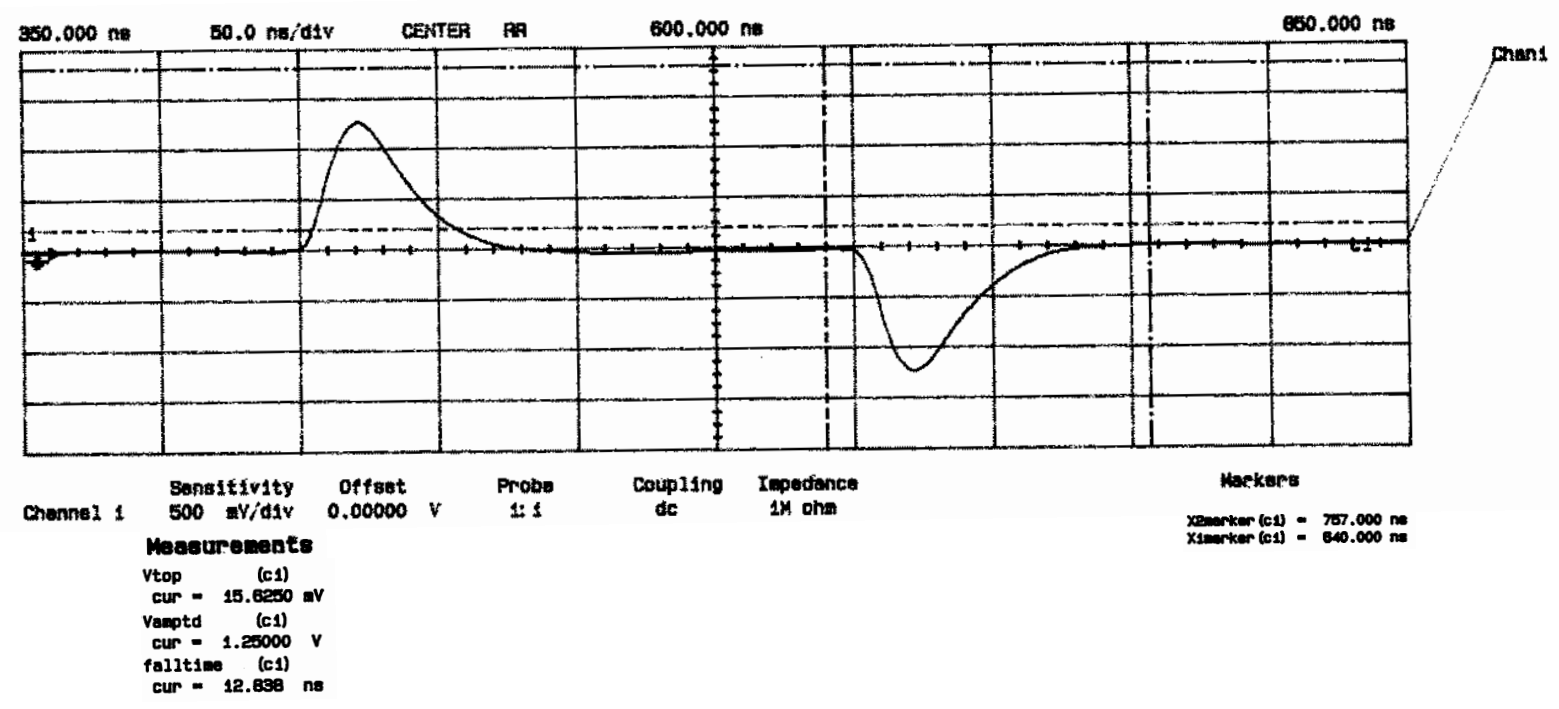

Figure 17: Signal after amplification and bipolar shaping $(\tau=20 \mathrm{~ns})$. The voltage modulation is a $200 \mathrm{~ns}$ square $40 \mathrm{mV}$ in amplitude. (test configuration according to figure 15). Scales are $50 \mathrm{~ns} / \mathrm{div}$ horizontally and $500 \mathrm{mV} / \mathrm{div}$ vertically. 


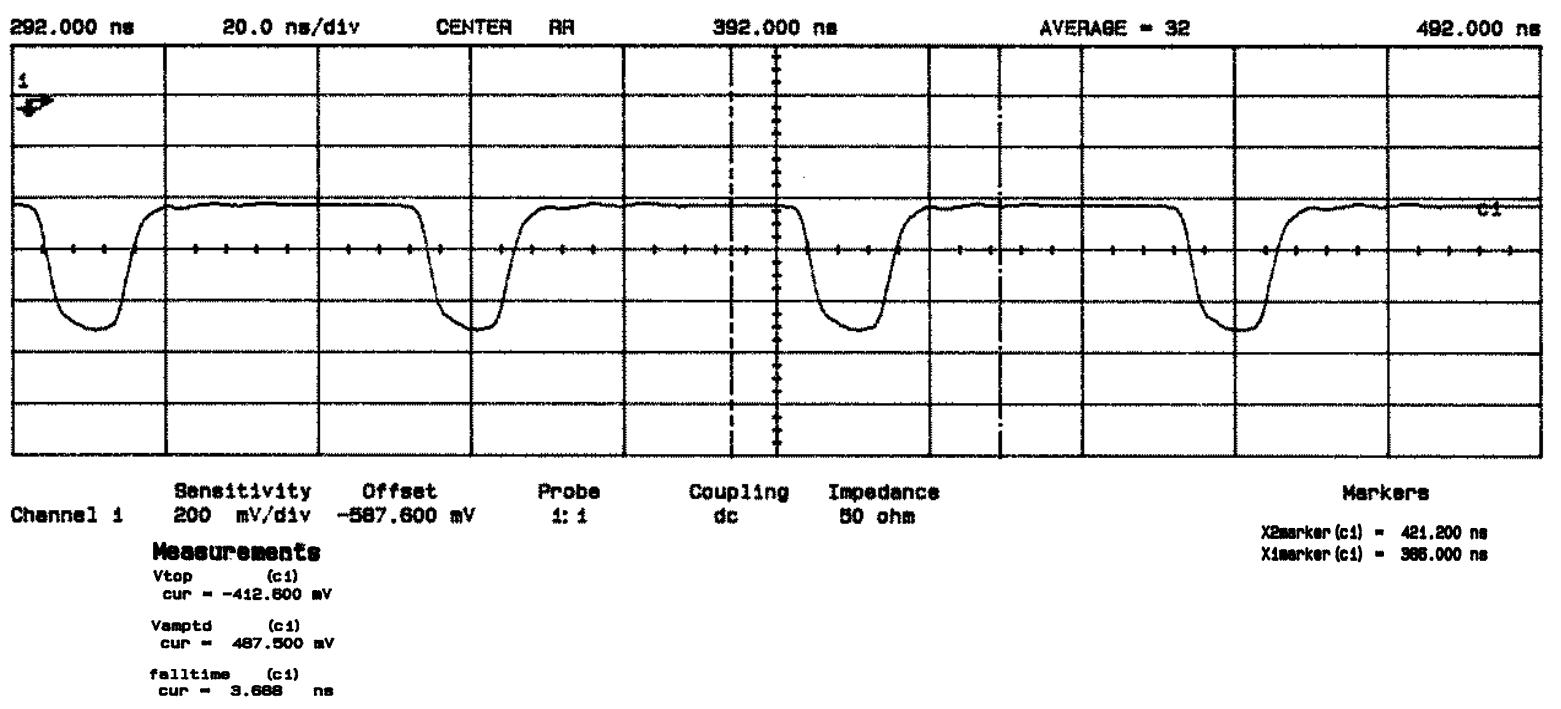

Figure 18: Response of the optical link to a 10 ns square modulation with a length of period of $50 \mathrm{~ns}$. The injected modulation has the same amplitude as for figure 16. The fall time (3.7 ns) and amplitude of the response $(485 \mathrm{mV})$ are not altered, which means the bandwidth of the system is larger than $100 \mathrm{MHz}$.

\subsection{Dynamic range and linearity of the optical links}

Figure 19 shows the dynamic responses of the eight channels from the second demonstrator, as measured in the conditions presented in the previous paragraph. The total range was split in several parts. The acquisition was driven by a user interface written with Labview. One parameter was the number of measurements for a given amplitude of impulse. From the highest part of the range down to $\sim 100 \mathrm{mV}$ pulses, the signal stood clearly above the noise and 10 measurements per point were taken (modifying this value had not significant effect). The lowest part of the dynamic was recorded with 50 measurements per point. The errors were computed online, and the RMS in ADC counts can be considered as an estimate of the noise. A linear fit was applied to each portion (in the lowest range, only the points 3 standard deviations above the noise were included in the fit, and the upper end of the range was determined as the point where the laser reaches saturation). Then pedestals were subtracted and the central portion $(100 \mathrm{mV}$ to $1 \mathrm{~V}$ pulses) was chosen as a reference (far from both noise and saturation levels). Other portions were scaled to the reference then a linear fit with zero as the origin was applied to the resulting curve.

Uniformity between the eight channels is good, which means the gain is almost the same for all channels, but the excessive noise for some channels prevented to explore the lowest part of the dynamic range. 


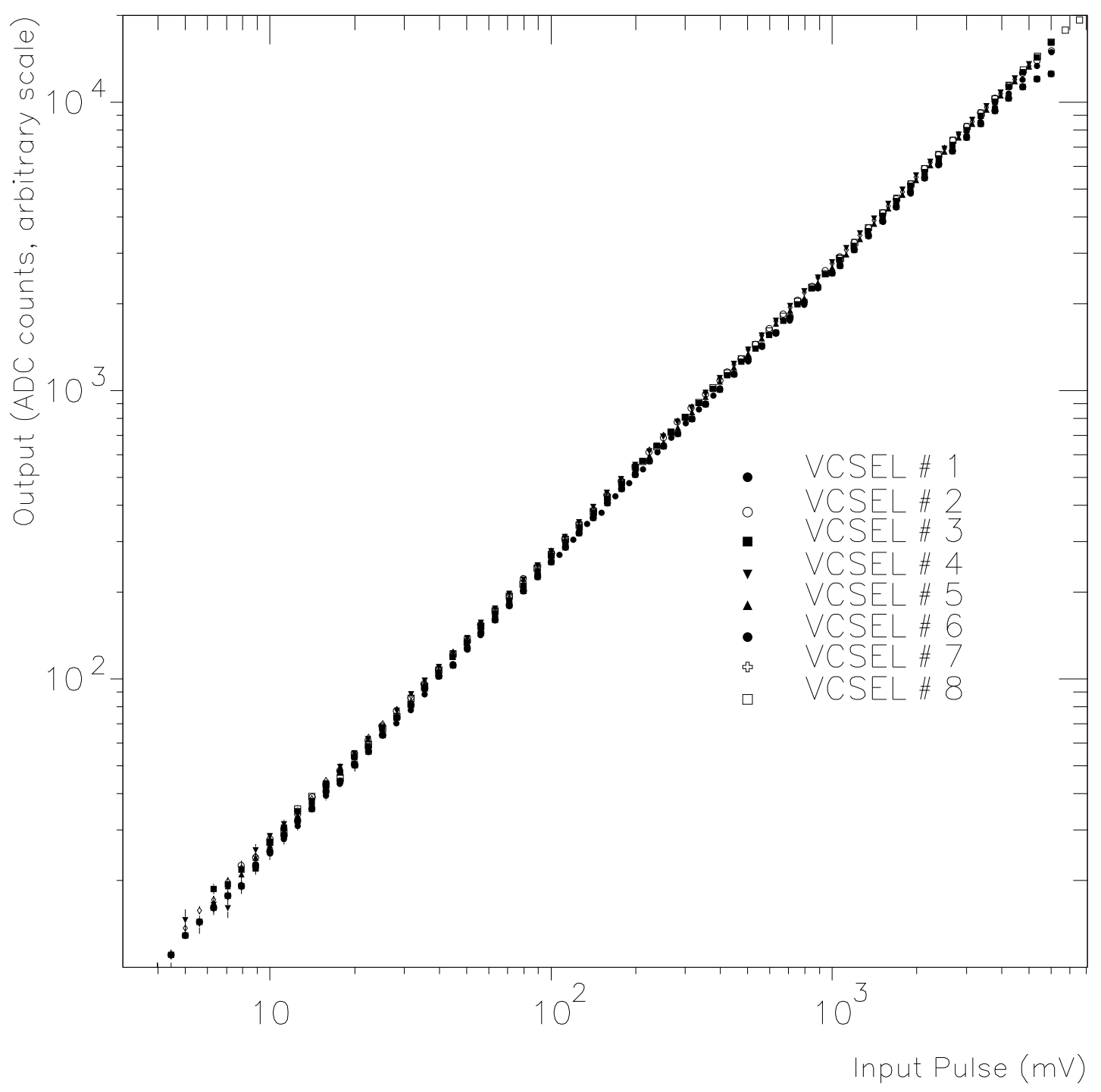

Figure 19: Dynamic response of the eight inverted polarity VCSELs, as a function of voltage modulation. 

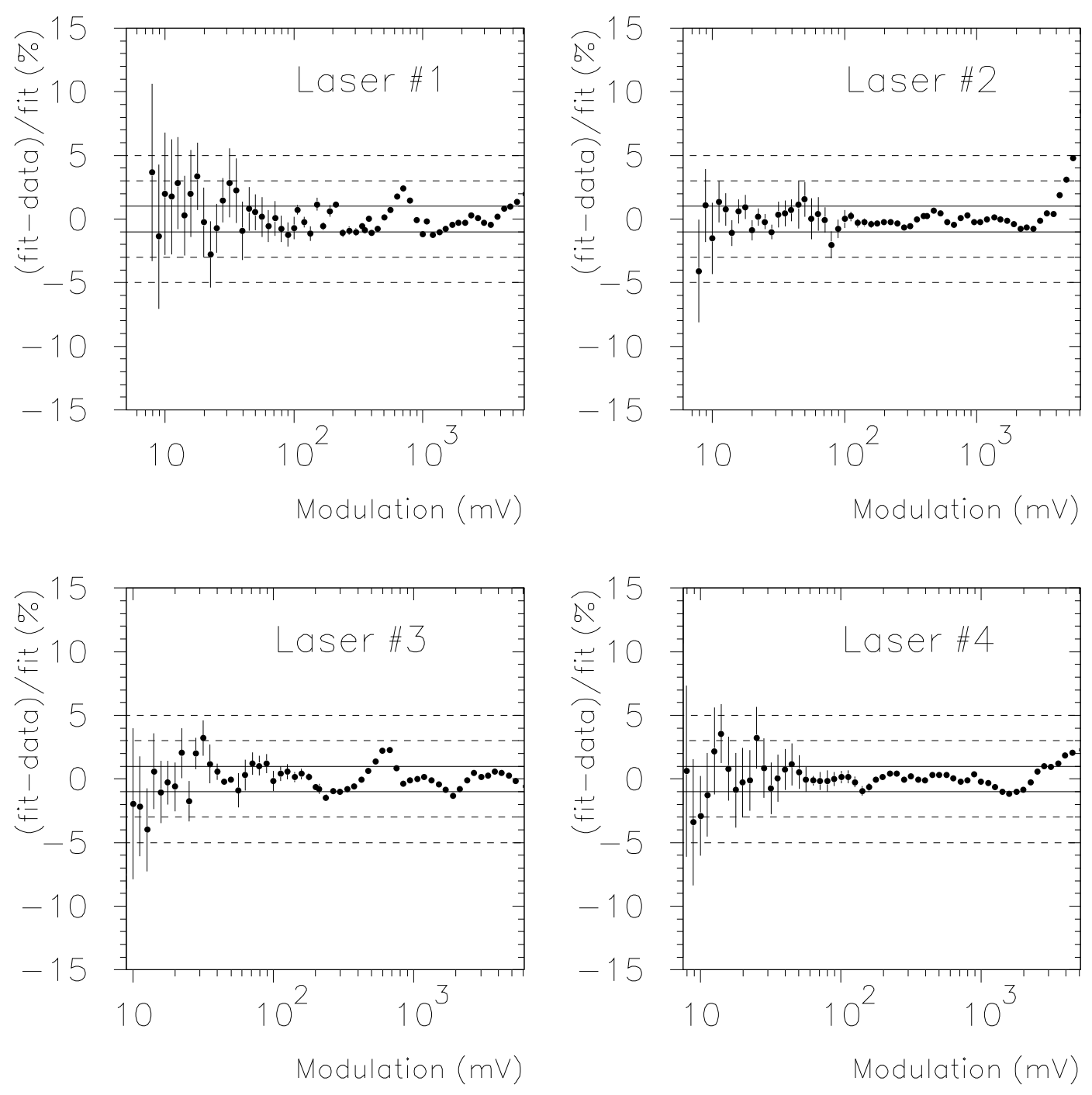

Figure 20: Non linearity for channels 1 to 4 . Levels at $\pm 1,3$ and 5\% are referenced. The loss of linear regime, most visible with the second laser, occurs for large signals and is equivalent to the thermal roll-off visible on static characteristics (e.g. Fig 3). 

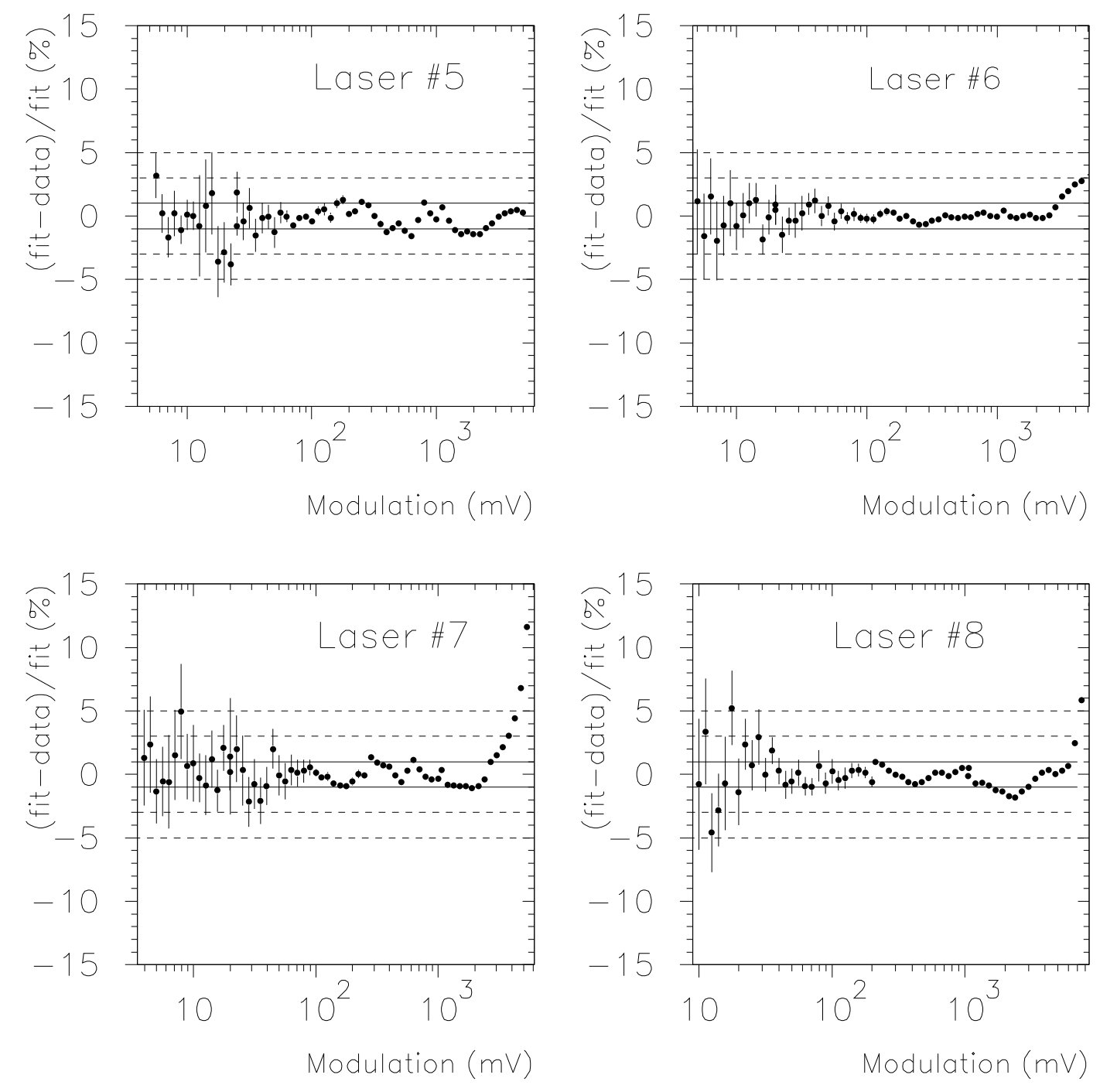

Figure 21: Non linearity for channels 5 to 8.

The same structure seems to appear for VCSELs 1 and 3 (voltage pulses between $500 \mathrm{mV}$ and $1 \mathrm{~V}$ ). It is not believed to be caused by a systematic effect from the read-out chain, as it is not visible for all lasers, and as non-linearities of the injection line and read-out chain, at the $1-2 \%$ level, have been corrected for.

Stability in time of the response of the optical link was then studied. Figure 22 shows the evolution of the response of channel 1 to a fixed value of modulation, along a period of twenty-three minutes ( 60 data acquisitions separated by 23 seconds each, 100 measurements per acquisition). Instabilities as high as $3 \%$ are visible.

Figure 23 shows that the read-out chain is very stable in time, at the level of better than $0.1 \%$. For this measurement the optical link was disconnected, and the pulse modulation used before was attenuated and fed to the $\mathrm{CRRC}^{2}$ amplifier (still 20 ns shaping time) whose output was read by the 12-bit ADC.

The level of instability measured can account for the patterns in figures 20 and 21 . Although it is not possible to decouple the responses of the emitter and receiver stages in 
our tests, the linear behaviour of the receiver seems well established [15], and the instability most likely originates in the optical emission.

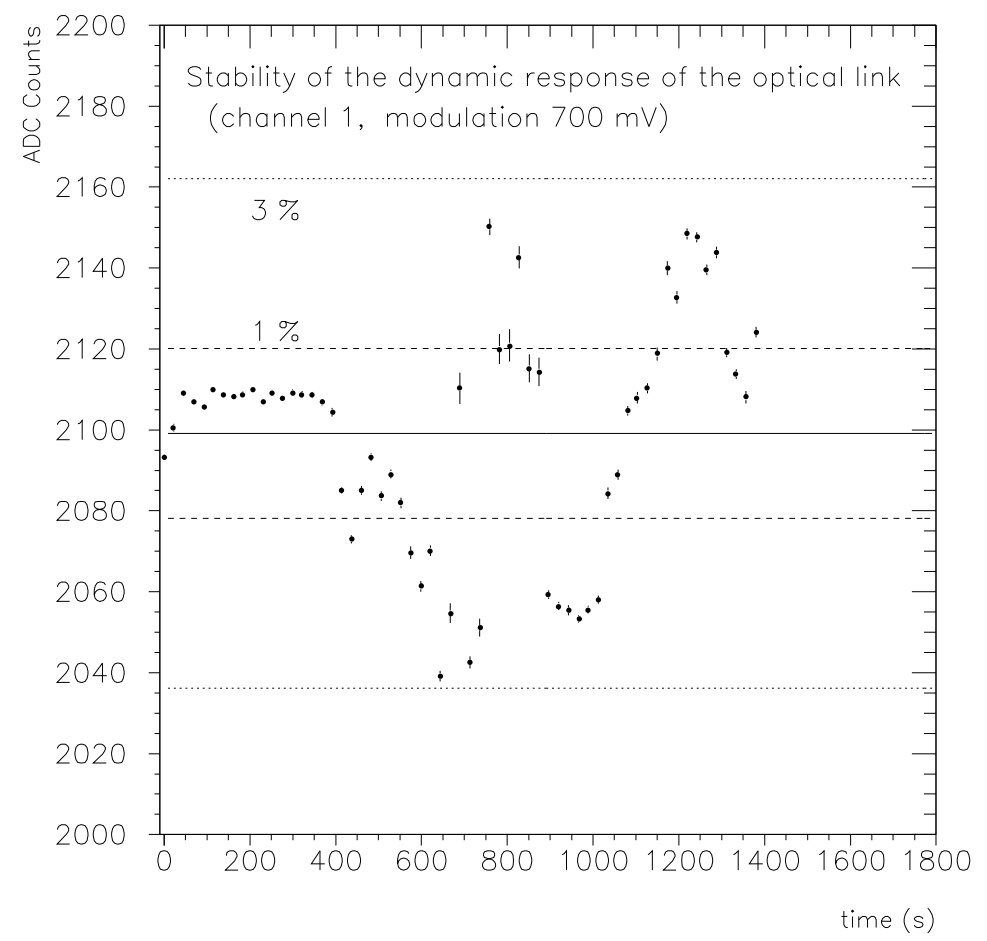

Figure 22: Long term stability of the optical link (response to a modulation of fixed amplitude).

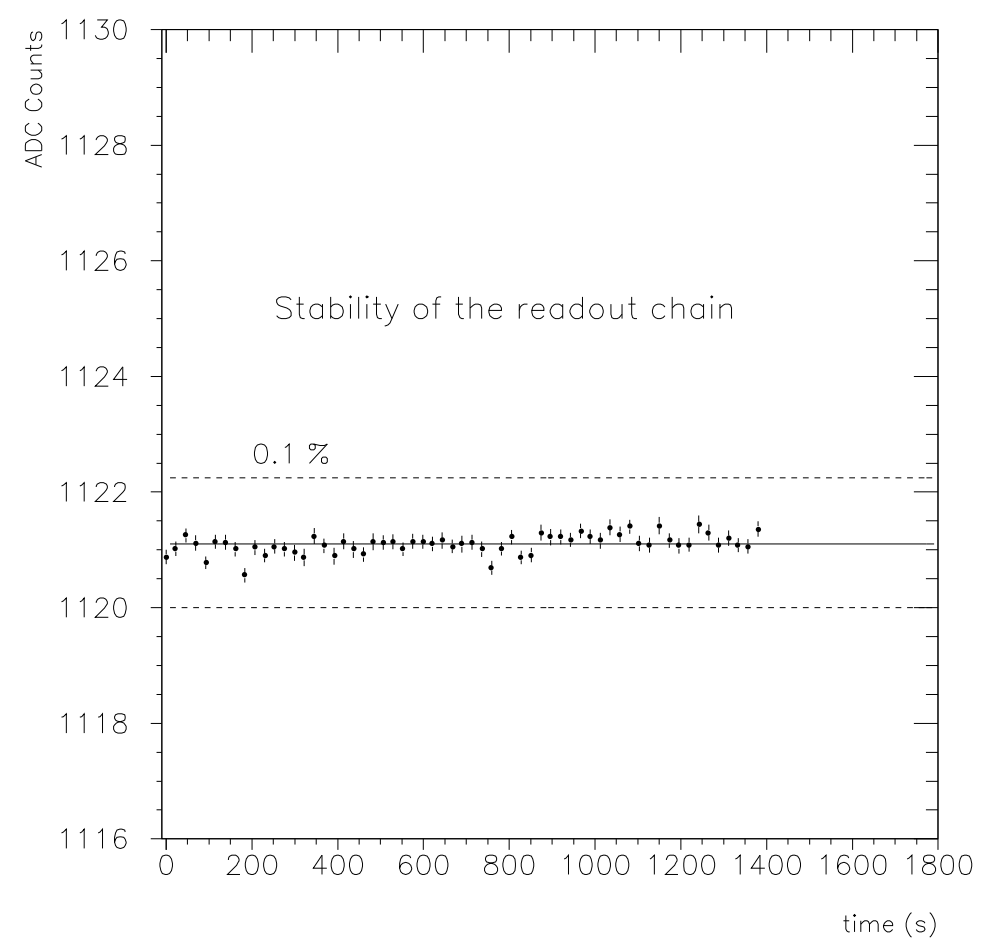

Figure 23: Long term stability of the readout chain used for the dynamic measurements (response to a modulation of fixed amplitude). 
Table 1 summarises the performance of each the eight channels, with the domain of linear fit, consequent dynamic range, and linearity.

\begin{tabular}{|c|c|c|c|}
\cline { 2 - 4 } \multicolumn{1}{c|}{} & Linear fit domain & Dynamic range & Deviation from linearity \\
\hline \hline Channel 1 & $9 \mathrm{mV}$ to $4800 \mathrm{mV}$ & $530(9$ bits $)$ & $\pm 4 \%$ \\
\hline Channel 2 & $7 \mathrm{mV}$ to $4200 \mathrm{mV}$ & $600(9.2$ bits $)$ & $\pm 4 \%$ \\
\hline Channel 3 & $10 \mathrm{mV}$ to $6000 \mathrm{mV}$ & $600(9.2$ bits $)$ & $\pm 4 \%$ \\
\hline Channel 4 & $8 \mathrm{mV}$ to $3200 \mathrm{mV}$ & $400(8.6$ bits $)$ & $\pm 4 \%$ \\
\hline Channel 5 & $5 \mathrm{mV}$ to $5000 \mathrm{mV}$ & $1000(10$ bits $)$ & $\pm 4 \%$ \\
\hline Channel 6 & $5 \mathrm{mV}$ to $3000 \mathrm{mV}$ & $600(9.2$ bits $)$ & $\pm 3 \%$ \\
\hline Channel 7 & $4 \mathrm{mV}$ to $3000 \mathrm{mV}$ & $750(9.5$ bits $)$ & $\pm 5 \%$ \\
\hline Channel 8 & $10 \mathrm{mV}$ to $6000 \mathrm{mV}$ & $600(9.2$ bits $)$ & $\pm 5 \%$ \\
\hline \hline
\end{tabular}

Table 1: Summary of the dynamic performance of the analogue optical link.

All but one channel for this second demonstrator show a dynamic range in excess of 9 bits, with non-linearities from 3 to $5 \%$. This confirms the performances of the first demonstrator [10], built with VCSELs of older generation.

\section{Conclusions}

We have built 8-channel demonstrators of analogue optical links based on VCSEL arrays, and tested them in view of their use for the read-out of the ATLAS liquid argon calorimeters.

The receiving end of the link is based on PIN photodiode arrays associated to custommade transimpedance amplifiers. It uses mastered techniques, and performs well within the specifications. Its industrialisation does not seem to raise any problem [15].

At the beginning of the project few emitters were known that could possibly meet our requirements in terms of compactness and optical performance. A prospective work was carried out, and led us to experiment with state-of-the-art VCSEL arrays. It must be noticed that the device used originated from development batches. The properties of such components are still subject to improvements.

Packaging issues turned out to be most problematic, as with a simplistic connector design the noise caused by optical feedback on the laser diodes limits the performance of the overall link to 9 bits in dynamic range and $5 \%$ in linearity.

The presented analogue link does not perform well enough to be used as is in the ATLAS experiment. There is evidence, though, that major improvements are within reach, provided a more efficient coupling of the emitters to the optical fibres is achieved. 


\section{References}

[1] B. Dinkespiler et al.,

An analogue optical link at liquid argon temperature, ATLAS Internal Note LARG-NO-08 (1994).

[2] B. Dinkespiler et al., Analogue optical links for the liquid argon calorimeters, Proceedings of the first workshop on electronics for LHC experiments, Lisbon, CERN/LHCC/95-56 (1995).

[3] ATLAS Liquid Argon Technical Design Report, CERN/LHCC/96-41.

[4] L.O. Eek et al., Development and performance of a receiver for an analogue optical link, ATLAS Internal Note LARG-NO-79 (1997).

[5] A. Rudge et al., A prototype LED/PIN diode optical link for the ATLAS semiconductor tracker, Nuclear Instruments and Methods A399 (1997) 119-128.

[6] H. Soda et al., GaInAsP/InP surface emitting injection laser, Jpn. J. Appl. Phys., 1979, Vol. 18, p2329

[7] R. Morgan et al., Vertical cavity surface emitting lasers come of age, Proceedings of the SPIE Vol. 2683, pp. 102-113, 1996.

[8] M. Lebby et al., Use of VCSEL arrays for parallel optical interconnects, Proceedings of the SPIE Vol. 2683, pp. 81-91, 1996.

[9] K. Lear et al., Vertical Cavity Lasers on p-doped substrates, Electronics Letters, Vol. 33, nํㅜ 9, 24 Avril 1997, pp 783-789

[10] B. Dinkespiler et al, Analogue optical links for the front-end read-out of the ATLAS liquid argon calorimeter, Proceedings of the third workshop on electronics for LHC experiments, London, CERN/LHCC/97-60 (1997).

[11] J. Beringer et al., Radiation hardness and life time studies of LEDs and VCSELs for the optical readout of the ATLAS SCT, ATLAS Internal Note INDET-NO-183 (1997)

[12] J. Guenter et al., Reliability of proton-implanted VCSELs for data communications, Proceedings of the SPIE Vol. 2683, pp. 102-113, 1996.

[13] D.L. Huffacker et al., Low threshold half-wave vertical cavity lasers. Electronics Letters, Vol. 30, pp 1946-1948, 1994.

[14] K. Lear et al., Life-testing oxide confined VCSELs: too good to last?, 
Proceedings of the SPIE Vol. 2683, pp. 114-122, 1996.

[15] L.O. Eek et al,

Development and performance of a receiver for an analogue optical link, ATLAS Internal Note LARG-NO-79, 1997.

[16] O. Hirota et al, Properties of intensity noises of laser diodes due to reflected waves from single-mode optical fibers and its reduction, IEEE Journal of Quantum Electronics, Vol. QE-17, nํㅜ 6, June 1981.

[17] K. Peterman and G. Arnold, Noise and distortion characteristic of semiconductor lasers in optical fiber communication systems,

IEEE Journal of Quantum Electronics, Vol. QE-18, nํㅜ 4, April 1982. 